\title{
Energy Heterogeneity Analysis of Heterogeneous Clustering Protocols
}

\author{
Shahzad Hassan and Maria Ahmad \\ Department of Computer Engineering, Bahria University Islamabad, Pakistan
}

\begin{abstract}
In Wireless Sensor Networks (WSN) the nodes have restricted battery power and the exhaustion of battery depends on various issues. In recent developments, various clustering protocols have been proposed to diminish the energy depletion of the node and prolong the network lifespan by reducing power consumption. However, each protocol is inappropriate for heterogeneous wireless sensor networks. The efficiency of heterogeneous wireless sensor networks declines as changing the node heterogeneity. This paper reviews cluster head selection criteria of various clustering protocols for heterogeneous wireless sensor networks in terms of node heterogeneity and compares the performance of these protocols on several parameters like clustering technique, cluster head selection criteria, nodes lifetime, energy efficiency under two-level and three-level heterogeneous wireless sensor networks protocols Stable Election Protocol (SEP), Zonal-Stable Election Protocol (ZSEP), Distributed Energy-Efficient Clustering (DEEC), A Direct Transmission And Residual Energy Based Stable Election Protocol (DTRE-SEP), Developed Distributed Energy-Efficient Clustering (DDEEC), Zone-Based Heterogeneous Clustering Protocol (ZBHCP), Enhanced Distributed Energy Efficient Clustering (EDEEC), Threshold Distributed Energy Efficient Clustering (TDEEC), Enhanced Stable Election Protocol (SEP-E), and Threshold Stable Election Protocol (TSEP). The comparison has shown that the TDEEC has very effective results over other over two-level and three-level heterogeneous wireless sensor networks protocols and has extended the unstable region significantly. From simulations, it can also be proved that adding node heterogeneity can significantly increase the network life.
\end{abstract}

Keywords: Heterogeneous wireless sensor networks, heterogenous, lifetime, clustering, cluster head, energy, nodes.

Received December 6, 2019; accepted February 1, 2021

https://doi.org/10.34028/iajit/19/1/6

\section{Introduction}

The term Wireless Sensor Network (WSN) can be defined as, "Integrating simple sensing, processing power, storage and communication capabilities into small-scale, low-cost devices and joining them into socalled wireless sensor networks" [1]. as shown in Figure 1 Boost in wireless networking technology, micro-manufacturing, integration, and embedded systems have spread modern generations of sensor networks desirable for a wide array of commercial, military and data centre applications [2, 3, 4]. WSN promises to inspire our way of living, interaction with the physical environment, and work.

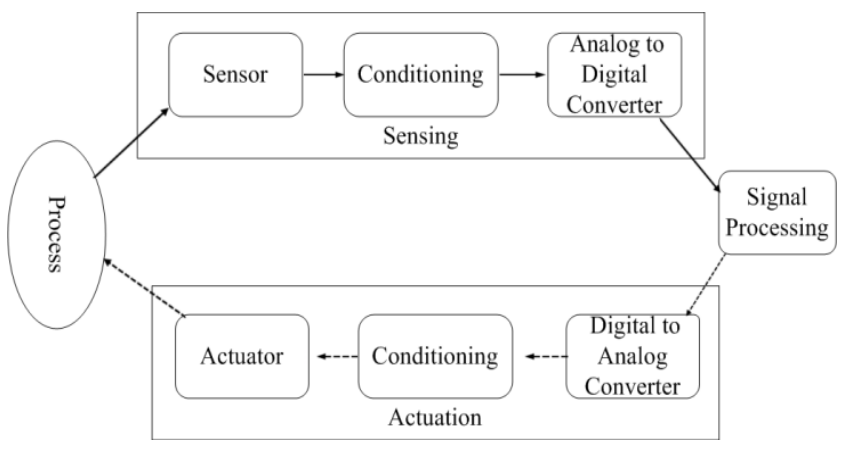

Figure 1. Data acquisition and actuation.

The WSN comprises an enormous number of sensor nodes ranging amongst few hundreds to thousands dispersed randomly through a geographic region or organized nearby to the phenomena. But network nodes have severe limitations in terms of limited energy, memory and transmission, and computation power [5]. Subsequently, the network nodes have restricted energy, which will result in energy depletion faster as a result the overall network lifetime will be reduced. To optimize the network life and management strategies certain goals like, prolonged network lifetime, scalability, coverage, and network simplicity are desirable, consequently, it is essential to propose an efficient and flexible network layer protocol $[6,7]$. To address above stated problems clustering protocols have been proposed by numerous investigators.

Clustering protocols offer the solution to exploit the nodes and network energy consistently to enhance the network lifespan time, maximize the packet delivery ratio and throughput as well clustering of nodes avoids long-haul communication of nodes with Base station (BS). Hassan et al. [9], authors presented low-energy adaptive clustering hierarchy, a pioneering benchmark on hierarchical clustering.

Low Energy Adaptive Clustering Hierarchy $(\mathrm{LEACH})$ was a revolutionary homogenous clustering protocol, in which nodes were equipped with equal uniform energy. In LEACH, the clusters are organized in each round and the cluster head selection takes place 
in every round which helps in the uniform energy utilization across the network. The $\mathrm{CHs}$ forwards the aggregated data received from the cluster members and forward it to the sink or base station. The [9] protocol lacks energy heterogeneity, there are energy variations to some threshold among these network nodes, and the nodes die out rapidly than a more uniform energy setting.

\section{Clustering}

To consume the network energy effectively and limit the correlated data, data aggregation and sensor fusion many novel techniques have been proposed by the Hassan et al. [10]. In the clustering technique, the nodes are partitioned into groups known as clusters. In general, one $\mathrm{CH}$ has been selected from the clusters and another cluster member connects with the cluster head. The cluster members communicate only with the cluster head and forward the sensed data to $\mathrm{CH}$. The $\mathrm{CH}$ then performs data aggregation and data fusion on the data obtained by the cluster members and transmits the fused data to the BS for further end-user processing.

Commonly the cluster is comprised of three main elements. Cluster Heads, Member Nodes, and the BS [8]. As shown in Figure $2 \mathrm{CH}$ selection is a major challenge to prolong energy efficiency and the network's lifetime. The $\mathrm{CH}$ is selected among the existing sensor nodes and the selection measures of $\mathrm{CH}$ vary in the suggested research but the key intention of these studies is to decrease the energy consumption and prolong the network life span.

The cluster member nodes or network nodes are those that are not elected as $\mathrm{CH}$ during the clustering selection phase. After the cluster selection phase, the nodes join the nearest $\mathrm{CH}$ forming a cluster. Due to the random placement of the nodes, sometimes the nodes are deployed very close to each other and their sensing region overlaps with others which results in highly correlated data.

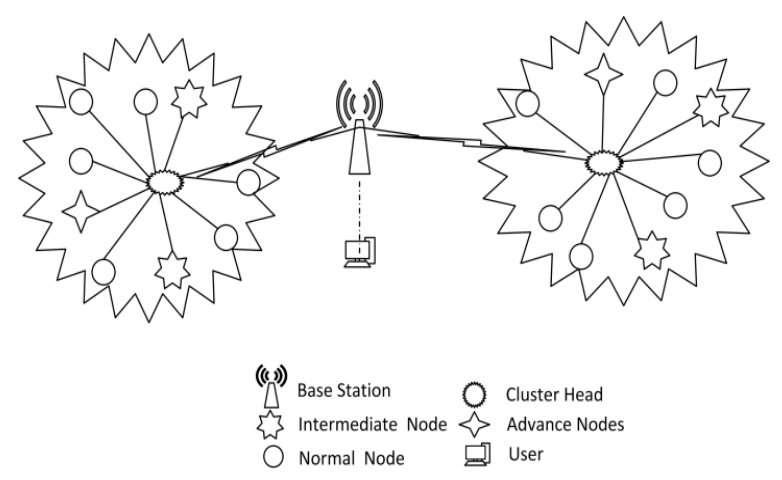

Figure 2. Clustering elements.

Designing the clustered network is the most crucial step for effectively utilizing the network energy. During the designing issue, certain aspects should be considered; like optimal cluster head size, CHs selection criteria, etc.

\section{Node Heterogeneity}

Based on numerous parameters on which Heterogeneous Wireless Sensor Networks (HWSN) can be categorized. For enhancement of network lifetime, node heterogeneity is exploited in many research areas. In heterogeneous sensor networks, the nodes are equipped with different capabilities such as different energy levels, sensing ranges, and computational power which are dependent on the application. The node heterogeneity benefits the HWSN in many ways e.g., decreases the latency, maximizes throughput, the minimal number of hops, and the end-to-end delivery is far greater concerning homogeneous WSNs [9]. Node heterogeneity can be divided into three kinds, i.e., Link heterogeneity, Energy Heterogeneity, and Computational heterogeneity as represented in Figure 3.

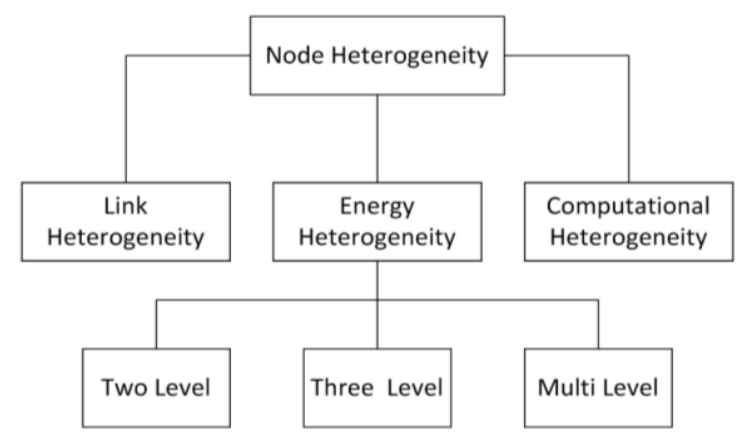

Figure 3. Node heterogeneity.

\subsection{Energy Heterogeneity}

In energy heterogeneity, the nodes are equipped with multiple energy levels. Usually, there are two types of nodes in the networks concerning the energy [9]. These are normal and advance nodes, and are often termed as two-tier HWSN protocols.

The advance nodes are more energized as compared to normal nodes and more probability is given to advance nodes to be selected as cluster head. In some research, the nodes operate in three-level energy heterogeneity i.e., normal, intermediate, and advance nodes, and are termed as three-tier HWSN protocols [10]. The intermediate nodes perform as a channel among the normal nodes and advanced nodes and their energy is kept between the advance and normal nodes.

\subsection{Computational Heterogeneity}

Computational heterogeneity refers to that the heterogeneous nodes are equipped with a powerful processor, high memory and can compute complex data [21]. It which ultimately reduces the processing latency as a result a maximum end-to-end delivery rate 
is achieved as compared to homogeneous sensor networks.

\subsection{Link Heterogeneity}

Link heterogeneity accounts for the reliable transmission distance between two nodes. To ensure reliable data transmission, link heterogeneity was implemented to meet the condition of maximum bandwidth and communication over long-haul distances [22].

\subsection{Two Level Node Heterogeneity}

The two-level HWSN model contains two kinds of nodes normal nodes $\mathrm{N}$ and advances nodes $\mathrm{m}$, equipped with different energy levels, where normal nodes are equipped with energy $\mathrm{E}_{0}$ and advance nodes are equipped with higher energy as related to normal nodes i.e., $\mathrm{E}_{0}(1+\alpha)$. Since the $\mathrm{N}$ is the total number of network nodes, then $\mathrm{Nm}$ is the sum of advance nodes while $\mathrm{N}(1-\mathrm{m})$ is the number of normal nodes. Therefore, the network's total initial energy is equivalent to the sum of energies of both types of nodes.

$$
\begin{gathered}
\mathrm{T}_{1 \mathrm{E} \_} \mathrm{N}(1-\mathrm{m}) \mathrm{E}_{0}+\mathrm{Nm}(1+\alpha) \mathrm{E}_{0} \\
\mathrm{~T}_{1 \mathrm{E} \_} \mathrm{N}_{\mathrm{E} 0}\left(1-\mathrm{m}+\mathrm{m}+\alpha_{\mathrm{m}}\right) \\
\mathrm{T}_{1 \mathrm{E} \_} \mathrm{N}_{\mathrm{E} 0}\left(1+\alpha_{\mathrm{m}}\right)
\end{gathered}
$$

The two-level HWSN [8] have $\alpha_{\mathrm{m}}$ times additional energy concerning homogeneous WSNs.

\subsection{Three Level Node Heterogeneity}

Three-level HWSN is equipped with three types of nodes, having different energy levels i.e., normal, intermediate, and advanced nodes. The energies of both normal and advanced nodes are the same as the two-level HSWN whereas the energy " $\mu$ " of the intermediate node is set among normal and advance nodes $E_{0}(1+b)$. Since $N$ is the sum of network nodes, then the total number of intermediate nodes becomes $\mathrm{N}_{\mathrm{bm}}$ and $\mathrm{Nm}$ (1-b) advance nodes. Therefore, in threelevel HWSN, the over-all initial network energy becomes,

$$
\mathrm{T}_{1 \mathrm{E} \_} \mathrm{N}_{\mathrm{E} 0}(1-\mathrm{m}-\mathrm{b})+\mathrm{mN}_{\mathrm{E} 0}(1+\alpha)+\mathrm{N}_{\mathrm{b}} \mathrm{E}_{0}(1+\mu)
$$

Where $\mathrm{b}$ represents the number of intermediate nodes equipped with energy $\mu$ and $\mu=\alpha / 2$. The three-level heterogeneous WSNs hold $(\alpha+\mu b)$ times additional energy concerning homogeneous WSNs. Enhanced Stable Election Protocol (SEP-E) and T- Stable Election Protocol (SEP) is the example of three-level HWSN.

\subsection{Multi-Level Node Heterogeneity}

In multi-level heterogeneous networks, the initial energy of nodes is arbitrarily dispersed over the close- set $\left[\mathrm{E}_{0}, \mathrm{E}_{0}(1+\alpha \max )\right]$, where $\mathrm{E}_{0}$ represents the initial energy and $\alpha \max$ represents maximum energy. Initially, the node the nodes are energized with $\mathrm{E}_{0} .(1+$ $\alpha_{i}$ ), which is $\alpha_{i}$ times additional energy. Therefore, the overall initial energy of the networks becomes

$$
\mathrm{E}_{\mathrm{T}_{\alpha i}}=\sum_{i=1}^{N} \mathrm{E}_{0}\left(1+\alpha_{\mathrm{i}}\right)=\mathrm{E}_{0}\left(N+\sum_{i=1}^{N} \alpha_{\mathrm{i}}\right)
$$

\section{Types of Hwsn Protocols}

\subsection{Types of Two-Level HWSN Protocols}

\subsubsection{SEP}

In [9] has introduced energy heterogeneity to prolong the stability period before the expiry of the first network node, which plays a critical role for certain applications in which the response from the network must be consistent. In [9] the cluster head selection is cantered on the weighted probability of individual nodes related to remaining energy. Since the advance nodes have additional energy which ensures that, this increment will work perfectly and the increased energy will have used efficiently, the advance nodes will elect cluster head more often than the normal nodes. The election of $\mathrm{CH}$ is made at the start of each round by choosing a random number $[0,1]$, if the value of the random number generated is less than the set threshold, the node will become $\mathrm{CH}$ in the current round.

Hassan et al. in [9] present a protocol that maximizes the stable region. As a result minimizes the unstable region and the protocol claims to improve the response of Clustered WSN in the occurrence of heterogeneous nodes. The downside of [9] is advanced nodes. Advanced nodes have extra energy and their probability to be selected as $\mathrm{CH}$ is higher concerning the normal node which results in higher energy depletion and at a certain stage the energy of them becomes equal to the normal nodes but still, the probability of advance nodes to be elected as $\mathrm{CH}$ is maximum.

\subsubsection{DTRE SEP}

A Direct Transmission and Residual Energy Based Stable Election Protocol (DTRE-SEP) [12] is an improved version of SEP. The proposed DTRE-SEP involves both direct transmission and residual energy of network nodes to form a new clustering protocol on similar fundamentals. The first improvement in [12] considers the transmission distance among the normal node and its cluster head and the distance between the normal node and BS. The second significant improvement has been made in the cluster head selection process. The probability of cluster head election is both weight and remaining energy-based.

\subsubsection{Z-SEP}

Zonal-Stable Election Protocol (Z-SEP) [13] is founded on zone partitioning. The network zones are 
separated into various zones i.e., zone 0,1 , and 2 . The normal node is arranged in network zone 0 while the advance nodes are positioned in network zone 1 and 2 . The normal nodes transmit directly to the base station and do not take part in the clustering process. The cluster head is selected form the zone 1 and 2. Though this protocol has enhanced the stability period of the network and minimized the instability period due to direct transmission the power of the normal nodes depletes very rapidly leaving a vast coverage region uncovered.

\subsubsection{ZBHCF}

In Zone-Based Heterogeneous Clustering Protocol (ZBHCP) [14] the network area is equally partitioned into four equivalent rectangular zones and identical numbers of nodes are randomly organized per zone. To restrict the energy consumption due to long haul communication between cluster head and nodes, each cluster head is elected from its corresponding zone by comparing its remaining energy with the zonal nodes.

The cluster head selection procedure is similar to [14] and cluster heads are randomly selected in each round of each zone. The selection of cluster heads is cantered on the randomly generated threshold value between 0 and 1 , if the produced number is maximum than the earlier set threshold the node will become cluster head in that specific round.

\subsubsection{DEEC}

Distributed Energy-Efficient Clustering (DEEC) [15] is a multi-level distributed clustering protocol that can also be operated in two-level heterogeneous networks. The election of cluster head is based on the ratio of residual energy of individual nodes and network average energy. This prior calculation of ratio consumes extra energy to share the info between the nodes.

The advance nodes having higher residual energy will be selected as cluster heads. This selection punishes the advanced nodes when their remaining energy is drained and becomes equal to the normal nodes. In this case, the advanced nodes tend to drains energy quickly.

\subsubsection{DDEEC}

A similar approach in [16] is adopted for estimating the average energy in the network and cluster head selection protocol is based on outstanding energy as applied.

When the energy level of normal nodes and advanced nodes reduces down to the edge of threshold remaining energy then both types of nodes use equal probability to be elected as $\mathrm{CH}$. Therefore, $\mathrm{CH}$ selection is balanced and more efficient. Threshold remaining energy " $\mathrm{Th}$ " is specified in [12] and given below:

\subsection{Types of Three-Level HWSN Protocols}

\subsubsection{SEP-E}

SEP-E [17] is a three-level heterogeneous protocol. SEP-E is an improved version of SEP that shows prolong network life period and exploits strength in the presence of energy heterogeneity.

In [17] an extra node has been added in the network termed as an intermediate node which acts as a connection between the normal node and advanced node, intending to reconcile and cater for multi-nodes diversity. The energy level of these nodes is kept between normal and advanced nodes. The energy of the intermediate node is kept between the normal and advance node.

The limitations of SEP-E are that the selection of cluster heads is probability-based and the selected $\mathrm{CHs}$ may be very nearby. Moreover, it does not take into account the enduring energy of the individual nodes thus the nodes that contain reasonably minimum residual energy can be elected as $\mathrm{CHs}$. This results in a degraded network lifetime. The $\mathrm{CH}$ may be deployed at the edge of the region or deployed in a low node density area. In this scenario, the energy utilization within a cluster be not uniform and the energy consumption will be high while communicating with the $\mathrm{CH}$. The stable region of SEP-E is marginally enhanced than SEP and the unstable region is considerably lower than SEP

\subsubsection{TDEEC}

Threshold Distributed Energy Efficient Clustering (TDEEC) [18] adopts the same cluster head election criteria and average energy consumption model [15] for better usage of network infrastructure.

\subsubsection{T-SEP}

Threshold Stable Election Protocol (T-SEP) [19] is a hybrid three energy levels reactive protocol that has implemented the characteristics of [17] and Energy Efficient Sensor Network protocol (TEEN) [20]. CH selection is done by the threshold value, due to three levels of node heterogeneity and being reactive network routing protocol, it produces increased stability period and network life span.

In [19] frequent data, the transmission is limited by threshold value and the transmission is done only in certain settings are met. To prolong the network lifetime [19] has used the features of [20] in which hard and soft threshold has been used.

The drawbacks of the [19] are, there is no calculation of energy levels for $\mathrm{CH}$ selection, and $\mathrm{CH}$ is still probability based in TSEP protocol. TSEP is Time-critical, another drawback of TSEP is, if a certain threshold is not attained, no data transmission will take place even if all the network nodes drain out their complete energy and the system will be remained 
unaware so TSEP is not suited for those applications where data transmission is required continuously

\subsubsection{EDEEC}

Enhanced Distributed Energy Efficient Clustering (EDEEC) [20] is an improved version of [15] which is a three-level heterogeneous protocol. EDEEC a threetier heterogeneous protocol. It contains three types of nodes, i.e., normal nodes, advanced nodes, and super nodes based on initial energy. The cluster head selection criteria are based on the [16].

\section{Simulation and Modelling}

\subsection{Performance Measures}

1. Stability Period: The Time Passed Meanwhile The Network Became Active Till The First Node Exhausts Its Energy.

2. Instability Period: The is the Time Interval That Starts as the First Node Dies out Till the Last Node in the Network Dies Out.

3. Network Lifetime: It is the Measure of the Time since the Network Becomes Active Till the Last Node Becomes Drains out Its Complete Energy.

4. Active Nodes: It is the Total Number of Nodes That Are Still Alive.

5. Dead Nodes: It is the Total Number of Dead Nodes Which Have Consumed Their Energy Completely.

6. Packets Transmitted to Base Station: It is the Total Sum of Packets Transmitted from the Cluster Head and the Nodes Transmitting Directly to the Base Station.

7. Number of Cluster Heads Per Round: It is the Total Number of Nodes That Are Selected as Cluster Heads in a Given Round.

\subsection{Simulations and Discussions for Two Level HWSN}

To further investigate, we have performed simulations on different clustering protocols under two-level heterogeneous WSN using MATLAB. The simulation parameters are given in table-1.100 nodes are randomly deployed in $100 \mathrm{~m} * 100 \mathrm{~m}$ area. For packet transmission, the energy model implemented in [9] is considered. For our simulations, we have selected ZBHCP, Z-SEP, DTRE-SEP, DDEEC, DEEC, and SEP for two-level heterogeneous WSNs. The simulated cases describe the number of active and dead nodes, the number of packets transmitted to the base station, and the number of the most critical parameters of cluster heads elected per round by setting parameters for $m$ which is the fraction of advanced nodes and $\alpha$ which is extra energy factor for advance nodes. Since in our case we are examining the energy heterogeneity we have considered only $\mathrm{m}=0.1$ and $\mathrm{a}=$ 1 . Rests of the parameters are given in Table 1 given below. We have performed independent simulations for each protocol.

Table 1. Network parameters.

\begin{tabular}{|c|c|}
\hline Parameter & Value \\
\hline Area & $100^{*} 100$ \\
\hline $\mathrm{n}$ & 100 \\
\hline $\mathrm{E}_{\mathrm{Ckt}}$ & $50 \mathrm{~nJ} / \mathrm{bit}$ \\
\hline $\mathrm{E}_{\mathrm{AD}}$ & $5 \mathrm{~nJ} / \mathrm{bit} / \mathrm{message}$ \\
\hline $\mathrm{E}_{0}$ & $0.5 \mathrm{~J}$ \\
\hline Packet Size & 4000 \\
\hline $\mathrm{P}_{\mathrm{opt}}$ & 0.1 \\
\hline$\varepsilon_{\mathrm{fs}}$ & $10 \mathrm{pJ} / \mathrm{bit} / \mathrm{m}^{\wedge} 2$ \\
\hline$\varepsilon_{\mathrm{mp}}$ & $0.0013 \mathrm{pJ} / \mathrm{bit} / \mathrm{m}^{\wedge} 4$ \\
\hline $\mathrm{d}_{\mathrm{NS}}$ & 87.70 \\
\hline
\end{tabular}

It can be observed from Figure 4 that in terms of stability period ZBHCP outperforms all other protocols. In ZBHCF network lifetime is enhanced by considering the intra-cluster communication and intercluster communication distance. Since the transmission is the main origin of energy depletion and an adequate amount of energy can be preserved by minimizing the transmission distance between the nodes. Since the nodes are have limited battery and the major portion of the energy is depleted in transmission which is directly proportional to transmission distance. Thus, minimizing the transmission distance between the nodes could significantly increase the network lifetime. The stable region of Z-SEP is improved than DTRESEP. In Z-SEP the cluster heads are elected from the advance nodes and the normal nodes do not participate in cluster head selection and transmit directly to the BS. Although this scheme has significantly enhanced the stable region of the network and decrease the unstable region due to direct transmission the energy of the normal nodes drain out very quickly leaving a huge coverage area uncovered. Since the normal nodes transmit data directly to the BS, and all of the normal nodes drain energy at the 2268th round leaving 60\% coverage area uncovered. DDEEC outperforms DEEC and SEP in terms of stable regions. In DEEC the sensor nodes with high initial and residual energy will have additional chances to be the $\mathrm{CH}$ than the lowenergy nodes. This choice reprimands always the advanced nodes, especially when their residual energy depletes and becomes in the range of the normal nodes. In this state, the advanced nodes die quickly than the others. While in SEP the $\mathrm{CH}$ selection probability of advance nodes is maximum as compared to normal nodes. Since in SEP the advance nodes are equipped with extra initial energy, thus the nodes with high initial will have more chances to be the cluster-heads than the low-energy nodes. To overcome the limitation of DEEC, DDEEC has introduced a residual energy threshold. When the energy level of advanced and normal nodes reduces to the limit of threshold residual energy then both kinds of nodes use the same probability to become cluster head. Therefore, $\mathrm{CH}$ selection is balanced and more efficient. The stable region of DEEC is greater than SEP, because, DEEC 
takes into account both the initial and residual energy, the stability period of DEEC is much longer than that of SEP.

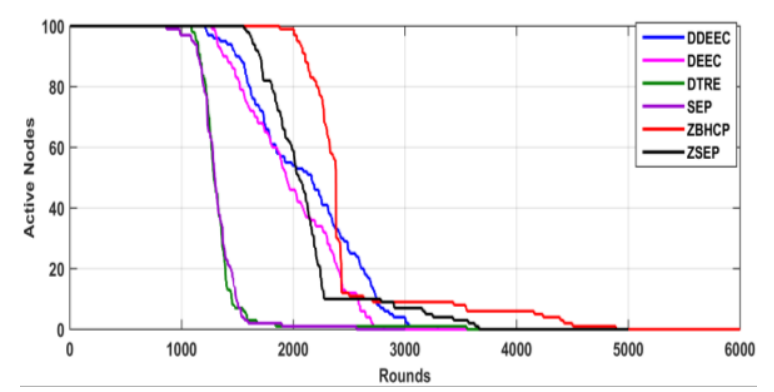

Figure 4. Comparison of active nodes per round.

Similar to Figure 5 we examine that the last node for ZBHCP, Z-SEP, DTRE-SEP, DDEEC, DEEC, and SEP drains out their energy at 4980, 3662, $3554,3295,2812$, and 2565 rounds respectively. The unstable region of $\mathrm{ZBHCP}$ is larger as compared to other protocols because the ZBHCP is a zone-based protocol that adopts inter and intra- clustered based communication and cluster heads are elected from the respective zones only which results in minimized communication range between the cluster heads and nodes.

The instability of Z-SEP is much larger than DTRESEP and DDEEC because the cluster heads are selected from the advance nodes and the number of cluster heads elected per round is minimum as compared to DTRE-SEP and DDEEC, since the fraction of advance nodes was $m=0.1$. However, the unstable region of the DDEEC is larger than DTRESEP, DEEC, and SEP.

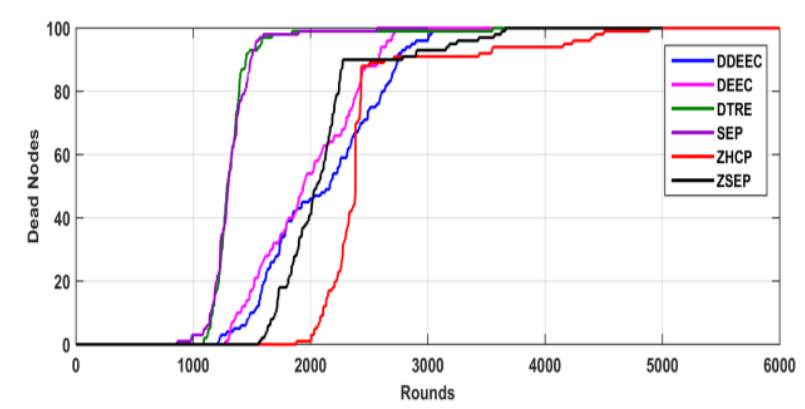

Figure 5. Comparison of dead nodes per round.

Figure 6 demonstrates the simulation results of packets transmitted to BS by ZBHCP, Z-SEP, DTRESEP, DDEEC, DEEC, and SEP. The throughput of the Z-SEP seems to be comparatively enormous as compared to other protocols.

In Z-SEP the $90 \%$ of normal nodes transmit directly to the base station. Since due to random deployment the communication radius of the nodes overlapped with each other and high correlated data is generated.

Since data aggregation is not performed at normal nodes due to which redundant data is transmitted to the base station [23]. The packets transmitted to the base station from DDEEC are higher than DEEC. The throughput of the SEP is very less as compared to other protocols.

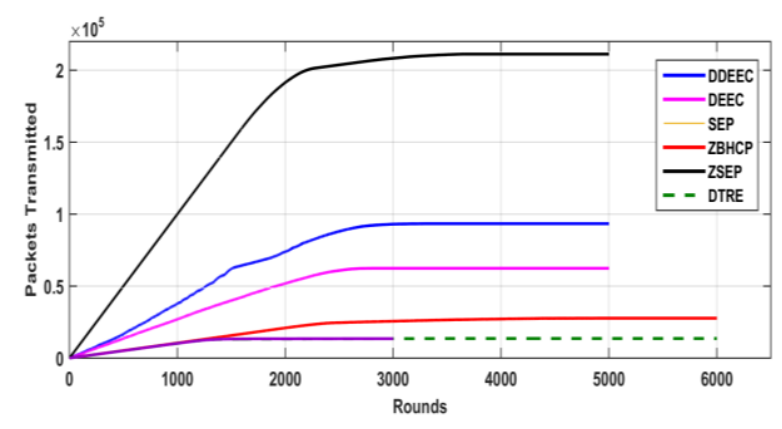

Figure 6. Packets transmitted to BS.

Figure 7 represents the number of cluster heads per round of ZBHCP, Z-SEP, DTRE-SEP, DDEEC, DEEC, and SEP. Due to enormous data size, the simulations are limited to certain rounds only. The number of cluster heads elected per round is the most crucial part of clustering protocols. If the number of cluster heads per round is not optimal the energy depletion will be high between the network nodes. In most rounds, no cluster head is elected and the criteria for the optimal number of cluster head election is not meet.

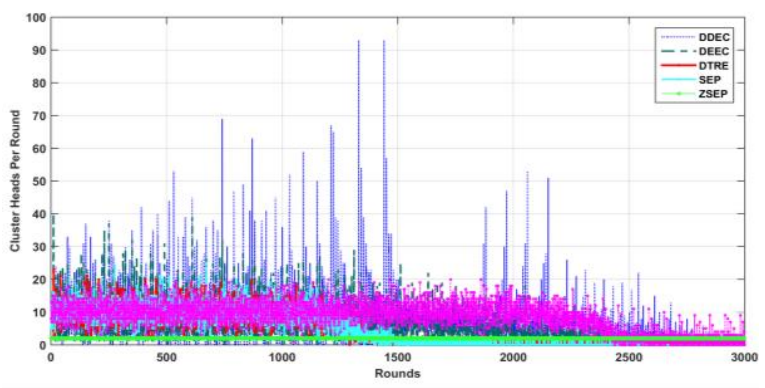

Figure 7. Number of cluster heads per round.

In ZBHCP the maximum number of cluster heads elected per round is 20. In Z-SEP the cluster heads are elected from the advance nodes so the number of cluster heads elected per round is 2, 22 in DTRE-SEP 93 in DDEEC, 40 in DEEC, and 25 in SEP. It is obvious the cluster selection criteria of the protocols become void as the number of nodes tends to die out.

\subsection{Simulations and Discussions for Three- Level HWSN}

The simulation procedure of the three-level HWSN is the same as the two-level. For our simulations, we have selected SEP-E, T-SEP, EDEEC, and TDEEC for three-level heterogeneous WSNs. Figure 8 shows the number of active nodes per round. It can be observed from the Figures 8 and 9 that TDEEC outperforms other protocols in terms of a stable and unstable region. The stability period of EDEEC, SEP-E, and T-SEP is lower TDEEC because the probabilities in TDEEC are defined separately for normal, intermediate, and advanced nodes. The stable region of EDEEC is 
improved than T-SEP and SEP-E. The number of active nodes in TDEEC is fairly greater than EDEEC because in TDEEC the equation of threshold used by nodes for cluster head selection is revised by including the remaining and average energy of that round. So, nodes having maximum energy will be selected cluster heads.

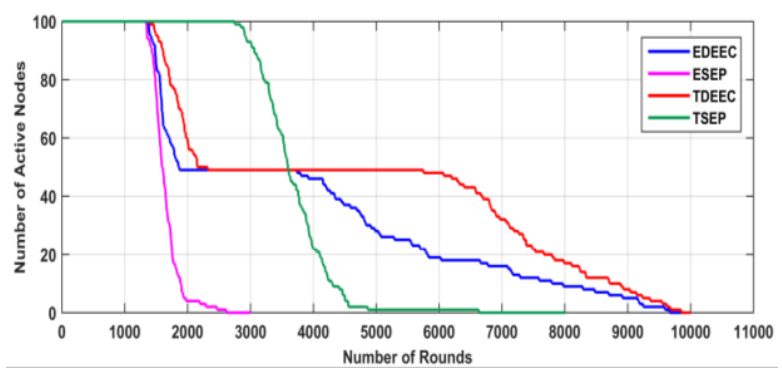

Figure 8. Number of active nodes per round.

Figure 9 reveals the number of dead nodes per round. Again, it can be concluded from the figure that the unstable region of the TDEEC is smaller than all protocols, while the unstable region of EDEEC is larger than T-SEP and SEP-E. In TDEEC the energy of the last node is exhausted at round number 9849, while in EDEEC at 9667, 6632 in T-SEP, and 2616 in SEPE.

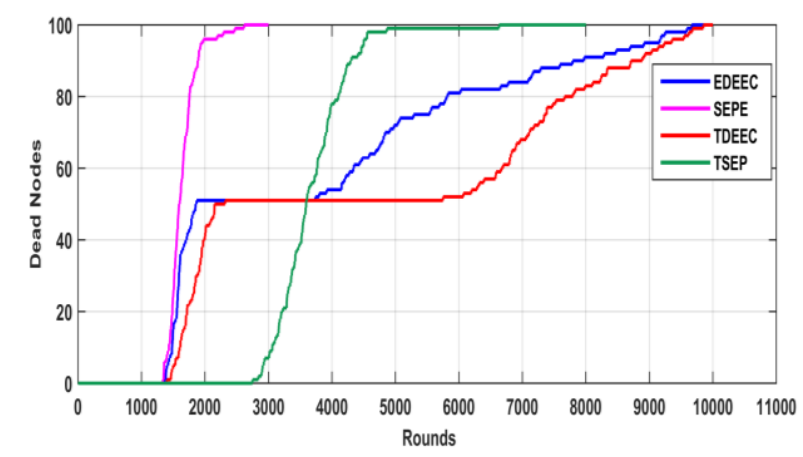

Figure 9. Number of dead nodes per round.

The number of packets transmitted to the base station is represented in Figure 10. The throughput of TDEEC and EDEEC is almost the same because they follow the same election probabilities. While the throughput of T-SEP is much larger than SEP-E.

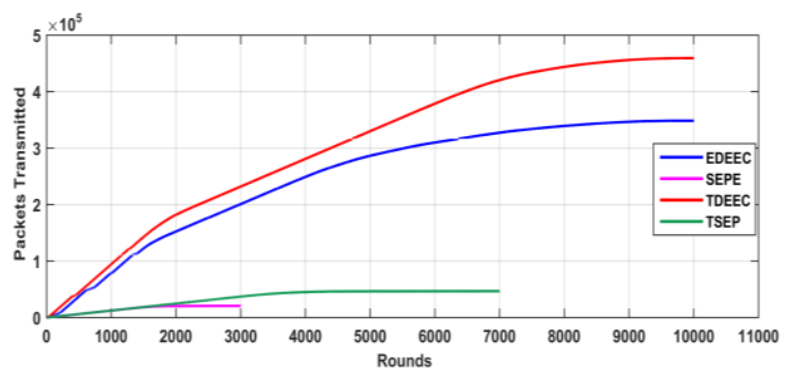

Figure 10. Packets transmitted to BS.

The number of cluster heads selected per round is shown in Figure 11. In SEP-E the highest number of cluster heads in a given round was 50, 52 in T-SEP.

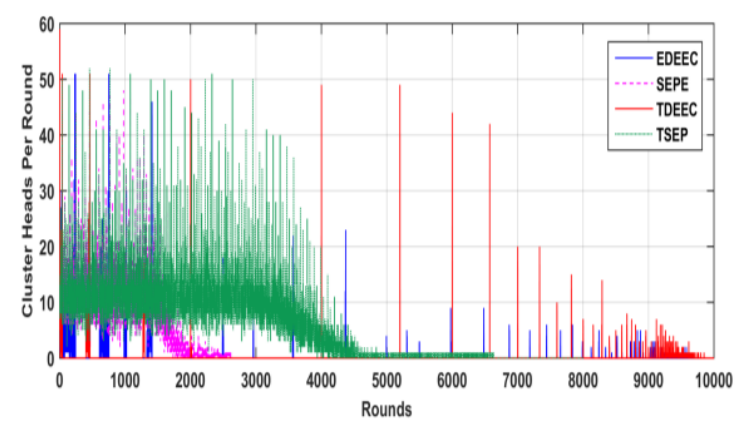

Figure 11. Number of cluster heads per round.

Table 2 states the number of rounds in which the first and last network node drains out their energy completely. It can be concluded that in ZBHCP energy is distributed more uniformly concerning the other protocols. The first node in ZBHCP, Z-SEP, DTRESEP, DDEEC, DEEC, and SEP exhausts their $1878^{\text {th }}, 1559^{\text {th }}, 1088^{\text {th }}, 1213^{\text {th }}, 1271^{\text {st }}$, and $926^{\text {th }}$ Rounds respectively while the last node of the network dies out at $4880^{\text {th }}, 3661^{\text {th }}, 3554^{\text {th }}, 3295^{\text {th }}, 2812^{\text {th }}$ and $2565^{\text {th }}$ round respectively. In the case of three-level heterogeneous protocols the first node in TDEEC, EDEEC, SEP-E, and T-SEP becomes deactivate at $1401^{\text {st }}, 1364^{\text {th }}, 1339^{\text {th }}$ and 2749 th rounds;

Table 2. Stable and unstable region.

\begin{tabular}{|c|c|c|}
\hline Protocol & 1st Node & Last Node \\
\hline \multicolumn{3}{|c|}{ Two Level HWSN } \\
\hline ZBHCP & 1878 & 4880 \\
\hline ZSEP & 1559 & 3661 \\
\hline DTRE-SEP & 1088 & 3554 \\
\hline DDEEC & 1213 & 3295 \\
\hline DEEC & 1271 & 2812 \\
\hline SEP & 926 & 2565 \\
\hline \multicolumn{3}{|c|}{ Three Level HWSN } \\
\hline TDEEC & 1401 & 9849 \\
\hline EDEEC & 1364 & 9666 \\
\hline SEP-E & 1338 & 2616 \\
\hline TSEP & 2749 & 6632 \\
\hline
\end{tabular}

The last node in TDEEC, EDEEC, SEP-E, and TSEP becomes deactivate at $9849^{\text {th }}, 9667^{\text {th }}, 2616^{\text {th }}$, and 000 rounds. As discussed above the TDEEC EDEEC, SEP-E and TSEP takes the advantage of additional nodes being added to the network, the last nodes which significantly enhance their unstable regions as compared to two-level protocols. From Table 2 it can be observed that ZBHCP has outperformed both two and three levels protocols in terms of the unstable region except for TSEP which is reactive in nature. This shows that prolonging the network lifetime in clustering protocols does not involve efficient cluster head selection algorithms, balanced cluster head but it should also account for the transmission distance between the network nodes.

Tables 3 and 4 shows the maximum number of elected CHs in a specific round. It can be verified from Tables 3 and 4 that none of the proposed protocols full fills the criteria of the optimal number of $\mathrm{CH}$ per round that should be around 10 [11] in a given round. 
Table 3. Highest number of CHs/round.

\begin{tabular}{|c|c|}
\hline Protocol & CHs \\
\hline Two Level HWSN \\
\hline SEP & 25 \\
\hline DTRE-SEP & 22 \\
\hline DDEEC & 93 \\
\hline DEEC & 40 \\
\hline ZSEP & 2 \\
\hline ZBHCP & 20 \\
\hline
\end{tabular}

Table 4. Highest number of $\mathrm{CHs} /$ round.

\begin{tabular}{|c|c|}
\hline \multicolumn{2}{|c|}{ Three Level HWSN } \\
\hline TDEEC & 60 \\
\hline EDEEC & 50 \\
\hline TSEP & 53 \\
\hline SEP-E & 50 \\
\hline
\end{tabular}

\section{Analysis and Discussions}

This article compares different heterogeneous protocols in terms of energy heterogeneity. The performance of the protocols was analysed by cluster head selection and selecting different performance parameters. Though the existing protocols have successfully enhanced the network lifetime still there are open issues that need to be addressed. Firstly, to the best of our knowledge none of the protocol addresses the node deployment issues since the heterogeneous wireless sensor networks contain three types of nodes normal, intermediate, and advance. In the above simulations, it was observed that due to random deployments of nodes, the intermediate and advance nodes were deployed very near to each other. Due to this random deployment, the nodes sensing radius overlap each other and enormous redundant data is collected.

A significant amount of energy is consumed in the data aggregation process. Random node deployment is the most frequently considered node deployment method in the HWSN. Though, it is inefficient from an energy efficiency perception due to Node's different energy levels. The unfeasibility usually arises in two sorts of conditions, one where the number of nodes is vast, and the other when the network is comprised of heterogeneous nodes i.e. nodes having various energy levels. In this scenario, the requirement of a welldesigned node deployment algorithm is becoming viable to maximize the network lifetime. Secondly the networks become unstable when the nodes tend to die and cluster head selection criteria become void. Since the energy depletion of cluster head is higher as compared to its member nodes due to multiple task data aggregation and data transmission. It can be seen in Figures 7 and 11 that the number of cluster heads selected per round is not optimal. If the number of cluster heads is not optimal per the round, the total energy consumption of the network increases exponentially which decreases the overall network lifetime.

\section{Conclusions}

Clustering has numerous advantages over traditional routing protocols and a substantial amount of energy can be preserved which is wasted indirect transmission or multi-hop communication. In clustering protocols, the nodes directly communicate with their cluster head thus eliminating the dependencies of other network nodes to forward their packets. It was argued that clustering has overcome major drawbacks faced in traditional routing. Further, that clustering protocols also minimizes the communication cost that occurred in the transmission of packets. The cluster head selection is application-specific it could be dynamic or static. Although clustering protocols offer many advantages clustering also suffers some drawbacks especially in $\mathrm{CH}$ selection. If the $\mathrm{CH}$ selection criteria are not appropriate and the number of cluster heads is not optimal, the network energy will deplete faster resulting in degraded performance of the network.

This article investigated mainly the nodes energy heterogeneity, the optimal number of cluster head per round of different protocols. In heterogeneous wireless sensor networks, cluster head selection plays a vital role in the enhancement of the network lifetime. If the number of cluster heads is not optimal per rounds, the network energy will deplete faster due to which the network life will be minimized and it was observed in some rounds that no cluster head was elected which show that no communication took place that rounds.

\section{References}

[1] Abbasi A., Jin H., and Wu S., "A SoftwareDefined Cloud Resource Management Framework," in Proceeding of Asia-Pacific Services Computing Conference, Bangkok, pp. 61-75, 2015.

[2] Abbasi A., Al-qaness M., Elaziz M., Hawbani A., Ewees A., Javed S., and Kim S., "Phantom: Towards Vendor-Agnostic Resource Consolidation in Cloud Environments," Electronics, vol. 8, no. 10, pp. 1183-1198, 2019.

[3] Abbasi A. and Hussain M., "A Qos Enhancement Framework for Ubiquitous Network Environments," International Journal of Advanced Science and Technology, vol. 43, pp. 37-48, 2012.

[4] Abidi W. and Ezzedine T., "Effective Clustering Protocol Based on Network Division for Heterogeneous Wireless Sensor Networks," Computing, vol. 102, no.1, pp. 413-425, 2020.

[5] Aderohunmu F. and Deng J., "An Enhanced Stable Election Protocol (SEP) for Clustered Heterogeneous WSN," Department of Information Science, 2009.

[6] Akyildiz I., Su W., Sankarasubramaniam Y., and Cayirci E., "A Survey on Sensor Networks," 
IEEE Communications Magazine, vol. 40, no. 8, pp. 102-114, 2002.

[7] Elbhiri B., Saadane R., Fkihi S., and Aboutajdine D., "Developed Distributed Energy-Efficient Clustering (DDEEC) for Heterogeneous Wireless Sensor Networks," in Proceeding of $5^{\text {th }}$ International Symposium on I/V Communications and Mobile Network, Rabat, pp. 1-4, 2010.

[8] Faisal S., Javaid N., Javaid A., Khan M., Bouk S., and Khan Z., "Z-SEP: Zonal-Stable Election Protocol for Wireless Sensor Networks," Journal of Basic and Applied Scientific Research, pp.1-9, 2013.

[9] Hassan S., Nisar M., and Jiang H., "DTRE-SEP: A Direct Transmission and Residual Energy based Stable Election Protocol for Clustering Techniques in HWSN," in Proceeding of International Conference on Communication Software and Networks, Chengdu, pp. 266-271, 2015.

[10] Hassan S., Nisar M., and Jiang H., "Energy Preservation in Heterogeneous Wireless Sensor Networks through Zone Partitioning," Journal of Electrical Engineering and Computer Science, vol. 2, no. 2, pp. 390-395, 2016.

[11] Heinzelman W., Chandrakasan A., and Balakrishnan H., "Energy Efficient Communication Protocol for Wireless Microsensor Networks," in Proceedings of the $33^{\text {rd }}$ Annual Hawaii International Conference on System Sciences, Maui, pp. 1-10, 2000.

[12] Hnaif A., Jaber K., Alia M., and Daghbosheh M., "Parallel Scalable Approximate Matching Algorithm for Network Intrusion Detection Systems," The International Arab Journal of Information Technology, vol. 18, no. 1, pp. 7784, 2021.

[13] Kashaf A., Javaid N., Khan Z., and Khan I., "TSEP: Threshold-Sensitive Stable Election Protocol for WSNs," in Proceedings of $10^{\text {th }}$ International Conference on Frontiers of Information Technology, Islamabad, pp. 164-168, 2013.

[14] Manjeswar A. and Agrawal D., "TEEN, A Protocol for Enhanced Efficiency In Wireless Sensor Networks," in Proceedings of $1^{\text {st }}$ International Workshop on Parallel and Distributed Computing Issues in Wireless Networks and Mobile Computing, San Francisco, pp. 189-192, 2001.

[15] Naeem M., Patwary M., and Maguid M., "Universal and Dynamic Clustering Scheme for Energy Constrained Cooperative Wireless Sensor Networks" IEEE Access, vol. 5 pp. 12318-12337, 2017.

[16] Qing L., Zhu Q., and Wang M., "Design of a Distributed Energy-Efficient Clustering
Algorithm for Heterogeneous Wireless Sensor Networks," Computer Communications, vol. 29, no. 12, pp. 2230-2237, 2006.

[17] Saini P. and Sharma A., "Energy Efficient Scheme for Clustering Protocol Prolonging the Lifetime of Heterogeneous Wireless Sensor Networks," International Journal of Computer Applications, vol. 6, no. 2, pp. 30-36, 2010.

[18] Saini P. and Sharma A., "E-DEEC- Enhanced Distributed Energy Efficient Clustering Scheme for heterogeneous WSN," in Proceedings of $1^{s t}$ International Conference on Parallel, Distributed and Grid Computing, Solan, pp. 205210, 2010.

[19] Sharma D., Ojha A., and Bhondekar A., "Heterogeneity Consideration in Wireless Sensor Networks Routing Algorithms: A Review," The Journal of Supercomputing, vol. 75, pp. 23412394, 2019.

[20] Smaragdakis G., Matta I., and Bestavros A., "SEP: A Stable Election Protocol for Clustered Heterogeneous Wireless Sensor Networks," in Proceedings of $2^{\text {nd }}$ International Workshop on Sensor and Actor Network Protocols and Applications, Boston, pp. 251-261, 2004.

[21] Sujith A., Dorai D., and Kamalesh V., "Energy Efficient Zone-Based Clustering Algorithm Using Fuzzy Inference System for Wireless Sensor Networks," Engineering Reports, vol. 3, no. 4, pp. e12310, 2020.

[22] Zhang T., Madhani S., and Berg E., "Sensors on Patrol (SOP): Using Mobile Sensors to Detect Potential Airborne Nuclear, Biological, and Chemical Attacks," in Proceedings of MILCOM IEEE Military Communications Conference, Atlantic, 2005.

[23] Zhang Y., Zhang X., Ning S., Gao J., and Liu Y., "Energy-Efficient Multilevel Heterogeneous Routing Protocol for Wireless Sensor Networks," IEEE Access, vol. 7, pp. 55873-55884, 2019. 


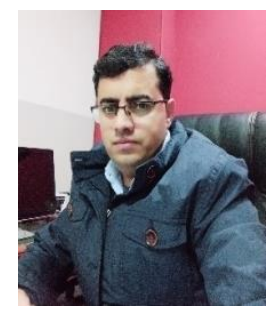

Shahzad Hassan is working as a HOD/Senior Assistant Professor in Bahira University Islamabad Since Nov 2016. He completed his BS. Computer Engineering from SSUET Karachi in 2007, MS Telecommunication Engineering from The University of Melbourne Australia and Ph.D. in Information and Communication Engineering degree from Huazhong University of Science and Technology, China. His research interests are WSN, Cloud Computing, VLC and IOT. He has more than ten years of teaching experience at undergraduate and graduate levels.

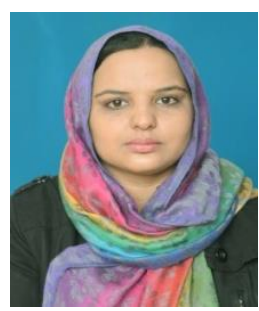

Maria Ahmad has obtained her BS.

Computer Engineering degree from CITT Wah (Campus) and MS. Electrical Engineering degree from Wah Engineering College, Wah. Currently she is enrolled in $\mathrm{PhD}$ Computer Engineering at Bahria University Islamabad, Pakistan. Her research interests are WSN and IOT. 